\title{
K-Nodes Multicasting Minimum Cost Spanning Trees in Wireless Mobile Ad Hoc Network (MANET)
}

\author{
Arun Kumar B. R \\ MCA Dept., Sir MVIT \\ Bangalore, Karnataka \& \\ School of Science \& \\ Technology \\ Dravidian University \\ Kuppam - A.P, India.
}

\author{
Lokanatha C. Reddy \\ Dept. of CS, School of \\ Science \& Technology \\ Dravidian University \\ Kuppam- A.P, India.
}

\author{
Prakash S. Hiremath \\ Dept. of P.G Studies \& \\ Research Group \\ Gulbarga University, \\ Gulbarga, Karnataka, India
}

\author{
Rajeswari Sheshadri \\ MCA Dept. \\ Sir MVIT, Bangalore \\ Karnataka, India
}

\begin{abstract}
Mobile Ad-hoc Networks (MANETs) are highly decentralized, independent and self-organizing networks. It is significant to study the cost of the network, to optimize the routing method by means of cross layer interaction across the layers of the network. In this paper, first we generate a minimum cost spanning tree for a given network of $\mathrm{N}$ - nodes using an efficient algorithm, and then we study the problem of constructing a K-node Multicast Minimum Spanning Tree (KMMST) for any given multicasting group with $\mathrm{K}$ nodes, where $\mathrm{K}$ is less than $\mathrm{n}$. Comparing the cost associated with the minimum spanning tree of the entire network with $\mathrm{n}$ nodes and the cost of KMMST, it is found that the cost of KMMST is significantly less compared to cost of the n nodes spanning tree.
\end{abstract}

\section{Keywords}

K-nodes multicasting, cost, spanning tree, routing.

\section{INTRODUCTION}

A mobile ad hoc network (MANET) is an unusual type of wireless mobile network which forms a transitory network without the support of an established infrastructure or a centralized administration. The MANETs has its applications which range from the civilian use to emergency rescue sites and in battlefield. The design and development of routing protocols in MANET is extremely challenging [1] as nodes can move arbitrarily, network topology can change frequently and unpredictably, and the bandwidth and battery power are limited. Many potential applications of Mobile Ad hoc Networks (MANETs) involve group communications among the nodes. Multicasting is a useful operation that facilitates group communications. This kind of application is efficient due to the broadcast nature of wireless network for it can improve the efficiency of the wireless links. As a result, multicast routing has become a hot area of research, much focused recently, and various multicasting protocols in MANET have been proposed.

A special form of multicasting known as broadcasting is the process in which a source node sends a message to all other nodes in MANET. Because of the ever-changing topology of MANETs, broadcasting is a primary communication primitive, essential to ad hoc routing algorithms for route finding and updating. Many protocols including dynamic source routing (DSR) [2], ad hoc on demand distance vector (AODV) [3], zone routing protocol (ZRP) $[4,5,6]$ and location aided routing (LAR) [7] use broadcasting to establish routes. The usual approach for broadcasting is through flooding. Flooding is appropriate for MANETs as it requires no topological knowledge.

Tree methods of multicasting in wired networks are a widely used technique. The research work in [8] has shown that the tree based method is an efficient, reliable and stable even in case of the ever changing network structure of the MANETs. The broadcasting using spanning tree [8] is done by forwarding a broadcast message not to all neighbors but only to those who are neighbors in the tree. Each message is received only once by each node as a tree is acyclic, contributes positively bringing enormous advantages over the existing methods.

The work in [13] computes the link disjoint minimum spanning tree for $\mathrm{n}$ nodes where $\mathrm{n}$ are the no. of nodes in the network. It has to be noted that it is not necessary to compute the spanning tree for all the $\mathrm{n}$ nodes always. Instead of that spanning tree can be computed to contain only multicast nodes of a particular multicast group.

A lot of research is done in MANETs related to the broadcasting and multicasting, for example [7-12] but efficient and scalable multicast routing in MANETs is a key issue which is less focused. The tree based approach has the benefit of high data forwarding efficiency. In highly mobile environments it leads to link broken failures. This failure can be prevented by selecting link stable spanning tree and continuing multicasting on alternate link stable edge disjoint spanning tree in case of first optimal tree fails [13] due to link failure, as in case of mesh based approach (high robustness in comparison with the tree based approach). The issue of scalability due to the large number of the nodes, which in turn affect the routing algorithms, needs to be studied. The parameters that need to be evaluated for scalability include no. of packets transmitted, total energy utilized to deliver packet from source to destination, frequency of determining routes between the nodes. This paper focuses on scalable, minimum cost multicasting in MANETs by computing spanning tree for $\mathrm{K}$ multicasting nodes set. We have presented and implemented a novel algorithm to compute the same.

The rest of the paper is organized as follows: In section 2, basic definitions are explained. section 3 brings out mathematical model of the study. Section 4 explains the general study of $\mathrm{K}$ nodes multicasting. The simulation study and result of $\mathrm{K}$ nodes 
multicasting spanning trees are focused in section 5 and finally it is concluded in section 6 .

\section{PRELIMINARIES}

We study a network where nodes communicate with their neighbors using wireless links. The following assumptions are made: The neighborhood of a node is the set of nodes which can receive a packet transmitted by the nodes. Any packet transmitted by a node is received by all its neighbors. The source node of a multicasting is believed to know the entire information essential to construct the multicast tree. We use the term edge and link interchangeably. The cost of an edge from $u$ to $v$ is same as $v$ to $u$ where (u, v) $\in \mathrm{E}$

Some of the important definitions of related to our discussion are given below.

- Complete graph: A simple graph in which every pair of distinct vertices is connected by an edge. The complete graph on $n$ vertices has $n$ vertices and $n(n-1) / 2$ edges, and is denoted by $K_{n}$. It is a regular graph of degree $n-1$.

- Connected graph: A graph $G$ is said to be connected, if for every pair of vertices u, v belongs to $G$, there exist a path, otherwise $\mathrm{G}$ is disconnected. A dis-connected graph has number of components; each component of a graph is a connected graph.

- Tree: It is a graph without cycles.

- $\quad$ Spanning Tree of a graph (SPT): It is a spanning sub graph that is a tree.

- $\quad$ Minimum Spanning Tree (MST) of a Graph $G$ : Among all the spanning trees of a weighted graph $\mathrm{G}$, the spanning tree whose sum of edge weights is least is called MST of a graph G.

- $\quad$ Edge Disjoint Minimum Spanning trees (EDMSTs): Let E1 and E2 be the set of edges of minimum spanning tree1(MST-1) and minimum spanning tree-2(MST-2) respectively, then MST-1 and MST-2 are said to be edge disjoint minimum spanning trees if E1 $\mathrm{E} 2=$ Null Set.

- $\quad$ K Node Multicast Minimum Spanning Trees (KMMST): A MST of graph G spanning K no. of multicast member nodes of the multicast group and forwarding nodes in $\mathrm{G}$ (FNG), FNG $\subseteq|V|-K$.

- $\quad$ Network Energy (NE (t)): Let $P N_{i}(t)$ be the no. of packets transmitted by the node $i$ at time $t$ and TE is the transmission energy required to transmit one packet. Let $\mathrm{n}$ be the no. of nodes in the network at time t. The energy expenditure made by the node $\mathrm{i}$ is the product of $P N_{i}(t)$ and TE. The energy of the network at any instant of time $t$ is the sum of energy expenditure made by each node of the network, that is, NE ( $t$ ) $=\sum_{i=1}^{n} P N_{i}(t)(T E)$.

- $\quad$ Nodes: All the nodes in the graph G.
- Members: All the members of the multicasting group.

- Non-members: The nodes which are not members of multicasting group.

- Forwarding nodes: All non-members which are part of the spanning tree helping to forward the multicast messages ( nodes with children)

- Leaf nodes: Member nodes which are at the end of a branch of a multicast tree( nodes without children)

\subsection{NETWORK MODEL}

A network is modeled as an undirected, connected and edge weighted graph $\mathrm{G}=\langle\mathrm{V}, \mathrm{E}\rangle$, where $\mathrm{V}$ is the vertex set and $\mathrm{E}$ is the edge set. A vertex can also be called as a node. An edge connecting any two vertices $\mathrm{u}$ and $\mathrm{v}$ is represented by $\mathrm{e}=(\mathrm{u}, \mathrm{v})$ and is also called as a link or an arc. Each link is associated with a link cost denoted by lc (e) $\geq 0$.

Given an undirected, connected and edge weighted graph $G$, the minimum spanning tree problem is to find a tree $\mathrm{T}=\left\langle\mathrm{V}, \mathrm{E}^{\mathrm{T}}\right\rangle$ where $\mathrm{E}^{\mathrm{T}} \subset \mathrm{E}$, with the minimum link cost.

Let $\mathrm{M}, \mathrm{F} \subseteq \mathrm{V}$ such that $\mathrm{M}$ is the Multicasting Group Set with $\mathrm{K}$ nodes and the $\mathrm{F}$ is the set of forwarding nodes for $\mathrm{M}$. It is clear from the definition of $\mathrm{M}$ and $\mathrm{F}$ that $\mathrm{M} \cap \mathrm{F}=\phi$. The $\mathrm{K}$ node Multicasting Minimum Spanning Tree (KMMST) problem is to find the minimum spanning tree for the set $\mathrm{M} \bigcup \mathrm{F}$.

If there are $\mathrm{p}$ multicasting group sets, say $\mathrm{M}_{1}, \mathrm{M}_{2} \ldots \mathrm{M}_{\mathrm{p}}$ for a given graph with their corresponding forwarding nodes set represented by $F_{1}, F_{2} \ldots F_{p}$ such that $M_{j} \cap F_{j}=\phi$, for $j=$ $1,2, \ldots p$ then each pair of $\left(\mathrm{M}_{\mathrm{j}}, \mathrm{F}_{\mathrm{j}}\right)$ gives rise to a unique minimum spanning tree $T_{j}$ for the set $M_{j} \bigcup F_{j}$.

The power set of a set $\mathrm{V} \in \mathrm{G}$ can be defined as the set of all subsets of $\mathrm{V}$. This includes the subsets formed from all the members of $V$ and the empty set. If a finite set $\mathrm{V}$ has cardinality $n$ then the power set of $\mathrm{V}$ has cardinality $2^{n}$. The power set can be written as $P(V)$. Further, $\mathrm{D} \subseteq \mathrm{V}$.

\section{STUDY OF K NODES MULTICASTING}

As already mentioned the scalability and cost in general of any adhoc network is an important parameters and needs cautious study. This is because as no. of nodes increases cost may also increase proportionately and protocols should be scalable.

We have considered connected random graph models consisting of $\mathrm{n}$ nodes which are connected by arbitrary no. of edges, selected randomly from the $n(n-1) / 2$ possible edges.

Lemma 1: Let $\mathrm{V}$ denote the set of all nodes in a given network and the cardinality of $\mathrm{V}$ be $\mathrm{n}$. Then the total number of multi casting group sets possible for the network is $2^{\wedge} n-(1+n)$.

Proof: Since cardinality of $\mathrm{V}=\mathrm{n}$, the Power set of $\mathrm{V}$, denoted by $\mathrm{P}(\mathrm{V})$ gives all possible subsets of $\mathrm{V}$ and $|\mathrm{P}(\mathrm{V})|=2^{\wedge} \mathrm{n}$. Since one of these $2^{\wedge} n$ subsets is the null set and there are $n$ subsets having exactly one node. These $1+n$ subsets do not contribute to multi casting since in multi casting group, we need at least 2 or more nodes for multicast communication. Hence the no. of subsets which contribute for multicasting are $2^{\wedge} n-(1+n)$.The analysis of the same is done and a graph is drawn and shown in the Figure 5, the subsection 5.2. 
If we are interested only in those members of $\mathrm{P}(\mathrm{V})$ which have minimum of 3 or more nodes then the possible multicasting group becomes $2^{\wedge} n-(1+n+n C 2)$ (see Figure 7 and Figure 9).

$$
\begin{aligned}
& \mathfrak{R}(V)=2^{n}-\left(1+n+n C_{2}\right) \text { where } \\
& n C_{2}=\frac{n !}{2 !(n-2) !} \ldots \ldots \ldots \ldots \ldots \ldots . . .(1)
\end{aligned}
$$

If we typically consider only the subsets of $\mathrm{V}$ containing exactly $\mathrm{K}$ nodes, then we are looking at the k-element subsets in $\mathrm{P}(\mathrm{V})$ and the number of which will be $\mathrm{nCk}$.

Lemma 2: The network energy of $\mathrm{K}$ node multicast minimum spanning tree of a given graph $G$ is less than or equal to the network energy of the spanning tree of $G$.

Proof: Let $\mathrm{G}(\mathrm{V}, \mathrm{E})$ be a graph with $\mathrm{n}$ nodes and $\mathrm{T}=\left\langle\mathrm{V}, \mathrm{E}^{\mathrm{T}}\right\rangle$, a spanning tree such that $\mathrm{E}^{\mathrm{T}} \subset \mathrm{E}$. Let $\mathrm{K}$ node multicasting minimum spanning tree (KMMST) be denoted by

$\mathrm{TK}=\left\langle\mathrm{VK}, \mathrm{E}^{\mathrm{T}}\right\rangle$ where $\mathrm{E}^{\mathrm{T}} \subset \mathrm{E}$. We know that $V K \subseteq V$, VK has two sets, a set of multicasting nodes $\mathrm{MG}$, $|\mathrm{MG}|=\mathrm{K}, \mathrm{K}<=|\mathrm{V}|$, that is $\mathrm{K}<=\mathrm{n}$ and a set of forwarding nodes, $|\mathrm{FNG}|<=|\mathrm{V}|-\mathrm{K}$. Therefore, $|\mathrm{VK}|<=\mathrm{K}+|\mathrm{V}|-\mathrm{K}$, that is,$|\mathrm{VK}|<=|\mathrm{V}|$. For a given network graph $\mathrm{G}=(\mathrm{V}, \mathrm{E})$, note that $\mathrm{K}$ node multicast spanning tree is a sub graph of spanning tree. By applying the definition of network energy of a graph,we can claim that network energy of KMMST of G <= MST of G. This is because no. of nodes in KMMST <=MST and hence energy required to transmit no. of packets also comparatively less in KMMST.

\section{SIMULATION STUDY AND RESULTS OF K NODES MULTICASTING}

In order to study the multicasting in particular cost, we have simulated the relevant aspects of ad-hoc network, and based on results we have drawn various graphs. The simulator system has been developed using JDK 1.5 version and compiled successfully in Windows XP service pack 2 having Intel processor of clock speed $1.5 \mathrm{GHz}, 256 \mathrm{MB}$ of RAM, VGA, and with other basic input devices.

The simulation process starts with a graph of 5 nodes and varied up to 50. The number of nodes and edges are fixed for each simulation. The arbitrary numbers of edges are selected and nodes are linked to form a connected network Then each link is assigned a cost whose value is assumed to be $>0$. Link cost from node $u$ to $\mathrm{v}$ is assumed to be same as $\mathrm{v}$ to $\mathrm{u}$. The example network with 10 nodes and 18 edges with link cost assigned are shown in the Figure 1. Its corresponding minimum spanning tree and $\mathrm{K}$ nodes spanning tree are shown in Figure 2 and Figure 3 respectively.

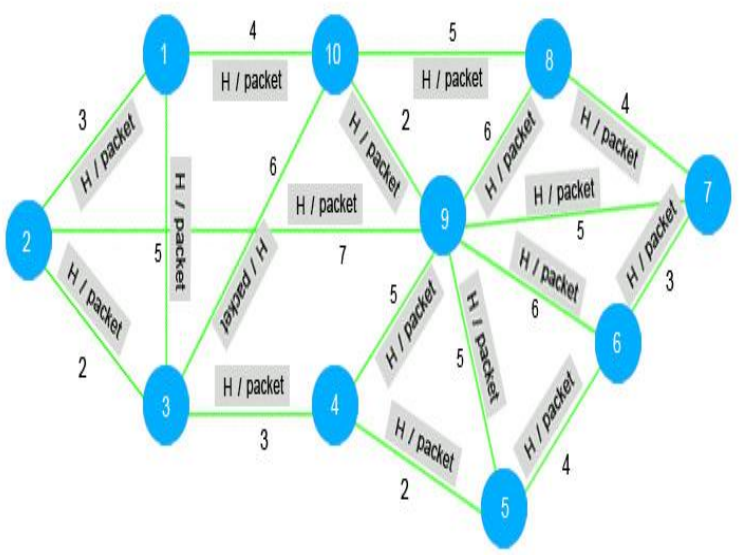

Figure 1. Example network $(\operatorname{cost}=77)$

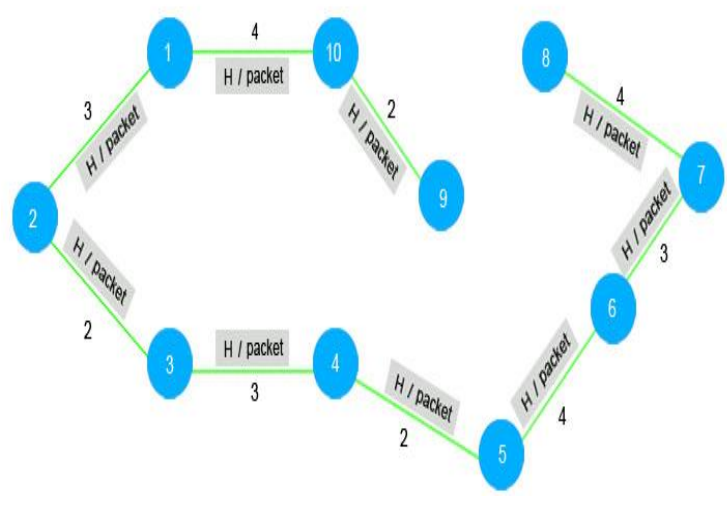

Figure 2: Minimum Spanning Tree $($ cost $=27)$

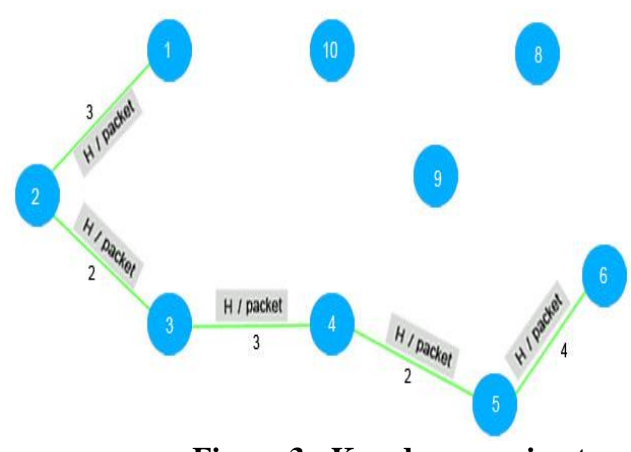

Figure 3: K nodes spanning tree

Where $|K|=6(\operatorname{cost}=14)$ 
The methodology adopted is as follows:

1. Choose $n$ nodes randomly and add the edges between them to form a connected network.

2. Compute Spanning tree on the entire network.

3. Compute $\mathrm{K}$ nodes spanning tree.

4. Compute cost of a graph, spanning tree and K nodes Spanning tree.

5. Compute the time taken to obtain cost of an entire network, spanning tree with $\mathrm{n}$ nodes and $\mathrm{K}$ nodes Spanning tree.

6. Repeat the above steps for number of experiments by varying no. of nodes.

7. Plot the graph of cost versus no. of nodes.

8. Plot the graph of computation time versus no. of nodes.

5.1 Algorithm to compute K nodes Multicast spanning tree

Input: Link Cost Adjacency(LCA) matrix of the given network.

1. The $N \times N$ link cost adjacency matrix $A=\mathrm{a}(\mathrm{i}, \mathrm{j})$ of $N \times N$ of a given network is with a( $\mathrm{i}, \mathrm{j})=$ link cost $(i, j)$, whenever two nodes (i,j) are connected by an edge and edge is associated with a link cost $>0$ ,otherwise zero.

2. Compute the shortest path from the given multicast source to each of the multicast destinations using Dijikstra's shortest path algorithm.

3. Construct the new link cost adjacency matrix based on the source and multicast destinations with costs $c$ $(i j)=$ cost of shortest path between $i$ and $j$.

4. Compute $K$ nodes multicast spanning tree applaying prim's algorithm on new LCA matrix.

5. Repeat the steps 2 to 4 for all the multicasting groups.

\section{Simulation Results}

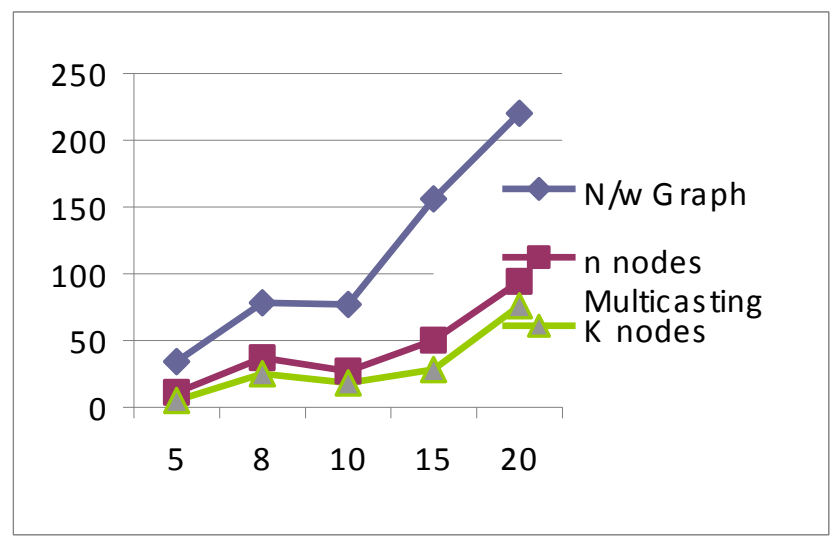

Figure 4: Graph of Cost Vs No. of Nodes

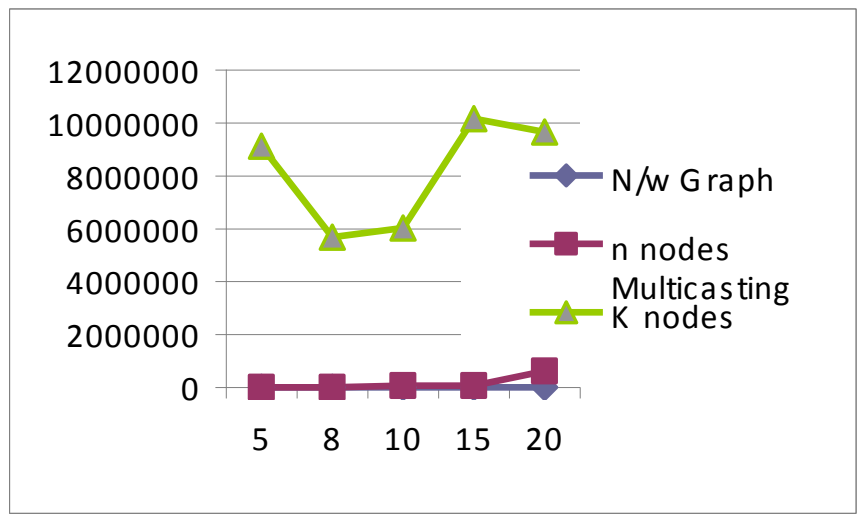

Figure 5: Graph showing time taken in nano seconds along $Y$ axis and no.of nodes along $X$-axis

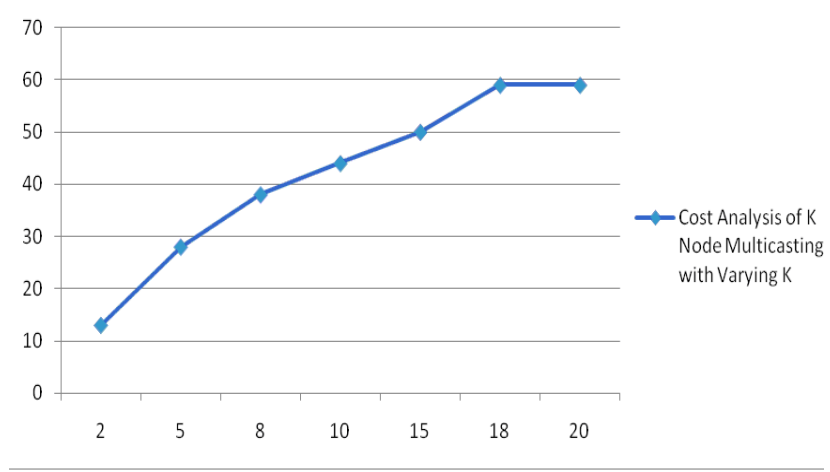

Figure 6: Cost Analysis of K Node Multicasting with Varying $\mathrm{K}$ from 2 to 20

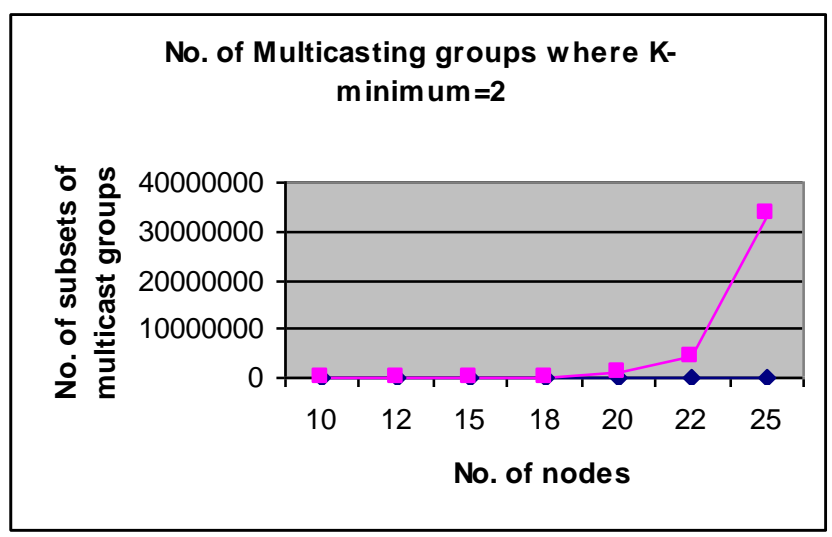

Figure 7: Analysis of multicasting groups where K- minimum $=2$ 


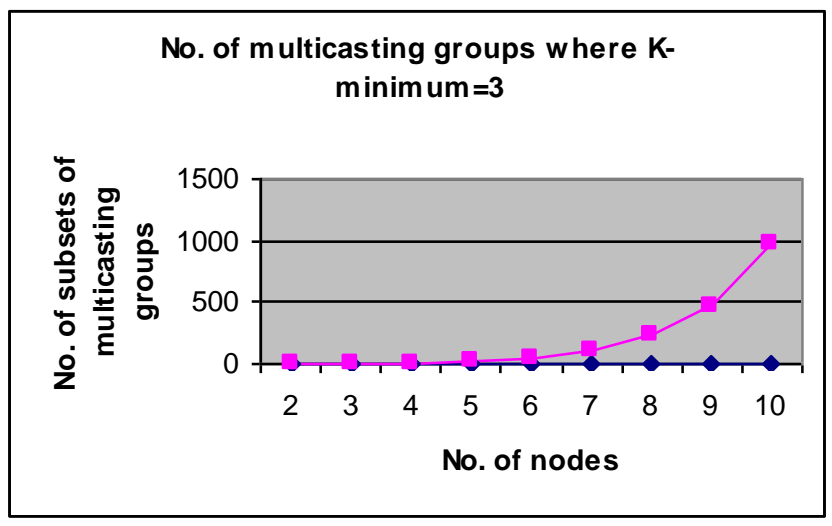

Figure 8: Analysis of multicasting groups where K- minimum $\mathbf{=} \mathbf{3}$

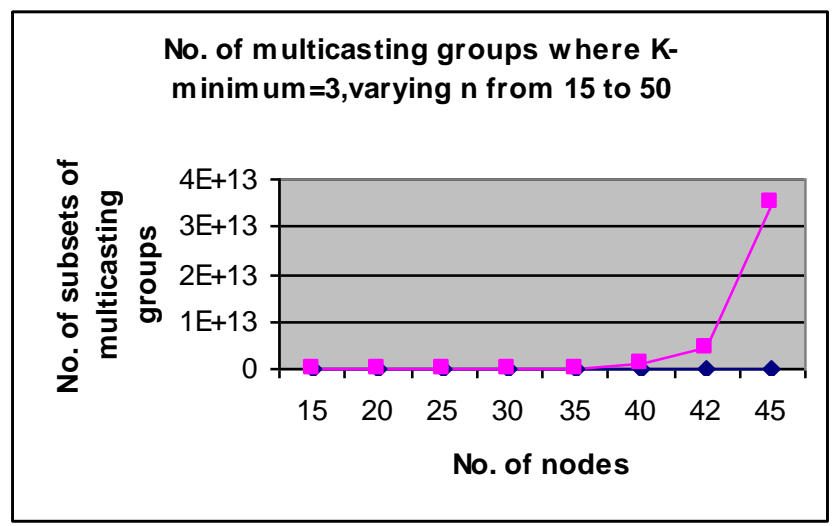

Figure 9: Analysis of multicasting groups where K- minimum $=3$, varying no. of nodes along $\mathrm{X}$-axis and no.of subsets of multicasting group ( $\mathrm{Y}$-axis)

\section{CONCLUSION}

In the environment of wireless ad hoc networks based on minimum spanning trees, broadcast trees and multicast trees are studied in depth. We claim that $\mathrm{K}$ nodes multicast trees in the context of MANETs is a novel study. In this paper we have introduced a novel algorithm and experimentally analyzed cost of $\mathrm{k}$ nodes multicast trees. The downside of the approach is that algorithm depends on source-multicast destination pair shortest path information, which may be costly or some times may not be possible to maintain the information in case of dynamic topology due to nodes mobility and more percentage of nodes are joining or and leaving the network.

\section{ACKNOWLEDGMENT}

All the Authors express their gratitude to all the authorities of Dravidian University, Kuppam, A.P, India for their extensive support. The first Author, Mr.Arun kumar B.R further extends his gratitude to SriKET, Dr.Viresh K Basalalli, Dr.M.S. Indira, Prof. R.H.Nagabhusan, of Sir MVIT, Bangalore, Karnataka for their constant support and encouragement.

\section{REFERENCES}

[1] D. Pan and Y. Yang, "FIFO-Based Multicast Scheduling Algorithm for Virtual Output Queued Packet Switches," IEEE Trans. Comput., vol. 54, no. 10, pp. 1283-1297, 2005.

[2] D. Johnson, D. Maltz and Y. Hu. The Dynamic Source Routing Protocol for Mobile Ad hoc Networks. Internet Draft: draftietf-manet-dsr-09.txt, 2003.

[3] C. Perkins, E. Beldig-Royer and S. Das. Ad hoc on Demand Distance Vector (AODV) Routing. Request for Comments 3561, July 2003.

[4] Z. Haas. A New Routing Protocol for Reconfigurable Wireless Networks.ICUPC 97, 562-566, 1997.

[5] Z. Haas and M. Pearlman. The Performance of Query Control Schemes for the Zone Routing Protocol. IEEE/ACM Transactions on Networking, 9(4):427-438, 2001.

[6] Z. Haas and B. Liang. Ad hoc mobility management with randomized database groups. Proceedings of the IEEE International Conference on Communications, 1756-1762, 1999.

[7] Y. Ko and N. Vaidya. Location-aided Routing (LAR) in Mobile Ad hoc Networks. Proceedings of the ACM/IEEE International Conference on Mobile Computing and Networking MOBICOM), 66-75, 1998.

[8] Alpr Jttner and dm Magi. Tree Based Broadcast in Ad hoc Networks. MONET Special Issue on WLAN Optimization at the MAC and Network Levels, 2004.

[9] Zeyad M. Alfawaer, GuiWei Hua, and Noraziah Ahmed, "A Novel Multicast Routing Protocol for Mobile Ad Hoc Networks", American Journal of Applied Sciences 4 (5): 333 338, 2007, ISSN 1546-9239, 2007 Science Publications.

[10] Penttinen, A et al, "Minimum cost multicast trees in ad hoc networks", Communications, 2006. ICC '06. IEEE International Conference, pp 3676 - 3681, June 2006.

[11] Blerta Bishaj, "Multicasting in ad hoc networks: Energy efficient", Helsinki University of Technology.

[12] Chao Gui and Prasant Mohapatra," Scalable Multicasting in Mobile Ad Hoc Networks", IEEE INFOCOM 2004.

[13] Arun Kumar B. R, Lokanatha C. Reddy, Rajan and Prakash S. Hiremath,"Route Failure Tolerant Multicast In Mobile Ad Hoc Networks Using Disjoint Minimum Spanning Trees", the second IEEE ICCSIT 2009, August 8 - 11, 2009, Beijing, China. 